\title{
Aerodynamic effects of corrugation configurations in sweeping- pitching flight
}

\author{
Haibin Xuan ${ }^{1}$, Jun $\mathrm{Hu}^{1}$, Yong $\mathrm{Yu}^{1,}{ }^{*}$ Jiaolong Zhang ${ }^{1}$ \\ ${ }^{1}$ School of Aerospace Engineering, Beijing Institute of Technology, Beijing 100081, P. R. China
}

\begin{abstract}
Some insects possess corrugated wings, which distinguish from the ordinary airfoils. It is important to research the corrugation effect on the aerodynamic performances. A series of corrugated wing models were designed based on former research in represent study to find out the underlying mechanisms. The effects of the corrugation pattern and inclination angle were studied using computational fluid dynamic (CFD) method during hovering flight at Reynolds numbers in the order of $10^{4}$. The instantaneous aerodynamic forces and the vorticity field around the wing models were provided. The findings are as follows: (1) the results of this paper show that patterns of corrugation have different effect on aerodynamic performances. The corrugated wing like Corrug- 1 changes the lift and drag very slightly compared with a flat-plate. The corrugation patterns like Corrug-2 and 3 of wing model reduce the lift and drag force. (2) the increase in the inclination angle has limited effect the aerodynamic forces. The inclination angles like corrug- 3 and 4 produce almost the same forces.
\end{abstract}

\section{Introduction}

Biomimetic inspires engineers to find easier and more practicable approaches to solve technical problems [1]. In recent years, the insect-like macro air vehicles (MAVs) are gaining popularity due to their potential application in environmental monitoring and surveillance and security [2]. Many insects have unique flying capabilities; insects can move forward, flap up and down, plunge and sweep, especially the dragonflies and the locusts. In addition, the wing corrugations of some kinds of insects have been seen one of the remarkable features, which show the advantages of low mass and high stiffness compared to smooth wings [3]. Extensive studies carried out by many researchers to research whether the corrugations benefit aerodynamic performances. These studies could be summarized as steady gliding flight and unsteady flapping flight.

Experimental studies conducted by Rees, Rudolph and Buckholz [4-6] showed that insects have both chordwise and spanwise corrugations to enhance the stiffness, but have limited effect on the production of aerodynamic forces in the condition of steady flow. Rees [4] used flow visualization method to identify the field features around a corrugated and smoothed envelope through the corner points of the corrugated section. He noted that flow-separation vortices trapped within the corrugation valleys compared to the smooth wing. Conversely, Kesel [7] compared different wing crosssectional corrugations of dragonfly with filled-smoothed airfoils, and pointed out that the corrugations of the dragonfly performs better than flat-plate wing. $\mathrm{Hu}$ and Tamai [8] conducted the experiment to investigate the flow behavior around a bioinspired corrugated airfoil compared with a traditional streamlined airfoil and a flat plate at the chord Reynolds number of Reynold number $(\mathrm{Re})=34000$ to explore the potential application of such bioinspired corrugated airfoils for micro air vehicle applications. They concluded that the corrugated airfoil has better performance over the streamlined airfoil and flat plate by preventing large-scale flow separation. Levy and Seifert [9] used (CFD) simulations to analyze the performances of corrugated and traditional smooth airfoil. They demonstrated that the corrugated airfoil outperformed the traditional streamlined airfoil when Re was in the range of 2000-8000. Chen and Skote [10] concluded that the profiled wing perform better than corrugated wing as the angle of attack (AoA) and the Re increasing for three dimensional gliding. Conversely, Bomphrey et al. [11] found that the corrugated wing generates marginally higher force coefficients than the smoothed wing when AoA up to $10^{\circ}$.

Meanwhile, researchers conducted simulations and experiment to investigate the effect of corrugation during unsteady flapping flight. Luo and Sun [12] studied ten representative insect wing models in sweeping motion with angle of attack $40^{\circ}$, the results of a corrugated wing model based on fruitfly and a smooth one showed that the corrugation had no effect on the aerodynamic forces. The reason might be that the separated flow or the size of leading-edge vortex exceeded the scale of the corrugation. Meng et al. [13] examined the aerodynamic effects of corrugation in insect wing models during hovering and forward flight using the method of CFD. They concluded that the time courses of aerodynamic

Corresponding author: yuyong@bit.edu.cn 
force of the corrugated wing are very close to those of the flat-plate wing.

Studies like above cited were mostly focus on the comparison of aerodynamic features between he corrugated and smooth wing or airfoil. The corrugation structures are little concerned. It can be seen that the effect of wing corrugation configurations for $3 \mathrm{D}$ simulations is still unclear, we performed 3D simulations using different corrugation configurations wing models during sweeping and pitching motion. The wing models used by former research $[14,15]$ to obtain deeper insights into the unsteady aerodynamics of flapping motion.

\section{Computational technique}

\subsection{The flapping kinematics}

It is common to simplify the laws of motion into mathematical function, two types of simplified laws, the trapezoidal and the sinusoidal are widely used. The idealized motion of 3D flapping wing consisted of pitching and sweeping [16], defined in Eqs. (1). The sketches of the pitching and sweeping motion profiles are shown in Fig. $1 b$.

$$
\begin{gathered}
\alpha(t)=90^{\circ}-\beta \sin (2 \pi f t), \\
\varphi(t)=\Phi \cos (2 \pi f t) .
\end{gathered}
$$

$b$ is set to $45^{\circ}$, the frequencies $f$ of the plunging and pitching motions are the same $(0.1 \mathrm{~Hz}), \phi$ has a value of $60^{\circ}$ in the present study. The flapping flat plate was built based on New et al.'s experiment.[17]. This wing model is assumed to be a rigid plate, with a thickness of $2 \%$ chord length. Fig.1a depicts the pitching and sweeping axis's locations.
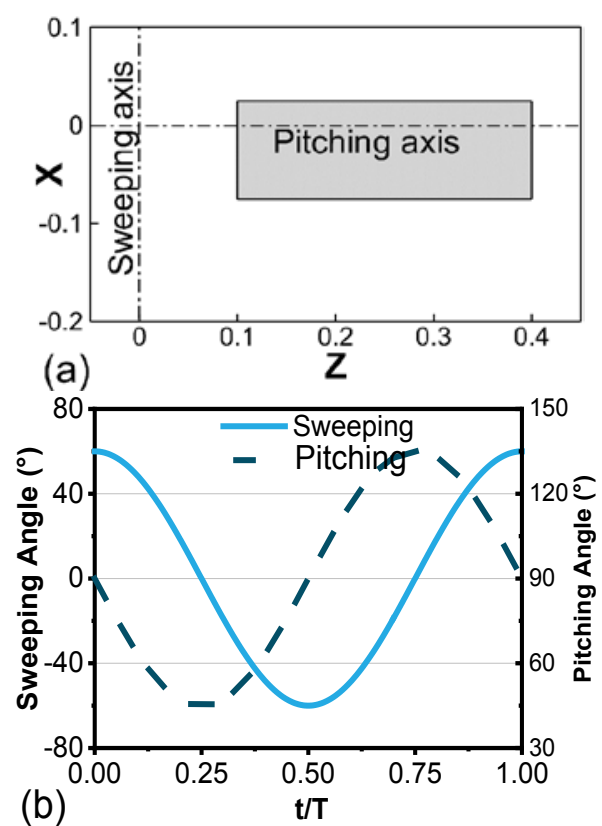

Fig. 1 Schematic of a) the pitching and sweeping axis locations and $b$ ) the sweeping and pitching motion profiles.

\subsection{Solver settings and computational domain}

Many literatures cited above stated that the airfoil corrugations cause the flow transition from laminar to turbulent. Levy and Seifert [9] stated that the flow pattern would be significantly different at higher Reynolds number and a transition from laminar to turbulent flow is expected. Hu and Tamai [8] stated that the corrugated wing that the protruding corners promote the transition of separated shear-layer flow from laminar to turbulent. The Reynold number in this paper is defined as: $R e=\rho U_{r e f} c / \mu, \rho$ is the liquid density, $c$ is the chord length, $\mu$ is the dynamic viscosity, the reference velocity $U_{r e f}=4 f \Phi R_{2}, f$ is the flapping frequency, and $R_{2}$ is the radius of second moment of area. Reynold number in this paper is in the order of $10^{4}$. This paper used the improved delayed Detached Eddy Simulations (IDDES) to perform simulations. The unsteady RANS models are employed in the boundary layer, while the LES treatment is applied to the separated regions.

The modified $\mathrm{k}-\varepsilon$ of the IDDES is defined as Eqs. (2)

$$
\begin{gathered}
\frac{\partial \rho k}{\partial t}+\nabla \cdot(\bar{\rho} \tilde{U} k)=\nabla \cdot\left[\left(\mu+\frac{\mu_{T}}{\sigma_{k 3}}\right) \nabla k\right]+P_{k}-\rho \beta^{*} k \omega F_{I D D E S} \\
F_{I D D E S}=l_{R A N S} / l_{I D D E S}
\end{gathered}
$$

The coupling of the pressure and velocity are handled using the coupled algorithm. The spatial discretization is carried out using a least square cell-based scheme. The pressure and momentum equations are solved with a second order scheme. All scaled residuals leveled off and reached $10^{-6}$.

In the present study, we used sliding mesh to model the motion of the flapping wing [18], a hybrid grid method is used to generate the grid. The domain size of the 3D wing model for flapping flight is essential for CFD simulations. The computational domain is $20 c$. The cell size was increased by keeping a factor of 1.05 away from the wing and 1.1 for the outer boundary. The outer boundary is set as a pressure outlet, the wing edge was set with the condition of no-slip wall.

\section{Results and discussion}

In this section, two sets of results flapping flight of corrugated wing models are discussed: 1) The effect of corrugation profiles was discussed first, the aerodynamic forces and the vorticity around the wing were provided to get deep insight into the corrugations. 2) Three different inclination angles based on a special wing model were discussed in detail. The aims of this section are to find out the effects of wing corrugations on flapping wing performance, and the contributions of different corrugated patterns.

\subsection{Effects of the corrugation patterns}

Before later discussion, it is important to define the aerodynamic characteristics. The lift coefficient $C_{L}$ and 
the thrust force $C_{D}$ are normalized using following equations:

$$
\begin{aligned}
& C_{L}=F_{L} /\left(0.5 \rho U_{r e f}^{2} S\right) \\
& C_{D}=F_{D} /\left(0.5 \rho U_{r e f}^{2} S\right)
\end{aligned}
$$

The grid and discretization time step influence the simulation results for unsteady simulations [19]. Three sets of grids were adopted to simulate for eliminating the mesh-error, this paper adopts the gird with $6 \times 10^{6}$ cells with a first layer of $0.0005 c$ after comparison. Small time steps are more likely to accurately describe physical phenomena, but increase the demanded resources of the simulation. In this study, it was verified through calculations that discretization time-step $\Delta t=T / 2000$ is the most suitable.

Fig. 2 shows the wing models used in this paper, the wing model is used in former research [20]. We modified the profile Corrug-1, replacing corrugated parts with smoothing plate. Two valleys and peaks were kept near the leading edge. Corrug-2 has the same valleys and peaks with Corrug-1 while has a camber rear part. Corrug-3 is covered with valleys and peaks. These four wing models have the same chord length and spanwise length.

Fig. 3 gives the time courses of lift and drag coefficients for three corrugated wings and one flat-plate wing in one flapping cycle. $T$ in the figure indicates flapping cycle. The fifth flapping cycle was used to avoid the non-fully developed flow field. Three significant observations could be made. Firstly, the $C_{L}$ differences of four wings are very small during downstroke while the differences are significant during upstroke. the second observation is that there is only very small difference between the results of different corrugated patterns. The last one is that $C_{L}$ and $C_{D}$ of flat-plate and corrug-1 wing models are mostly the same and symmetrical. The results presented in Fig. 3 show that the force coefficients were significantly asymmetric during the upstroke and downstroke for Corrug-2 and Corrug-3 (shown in Fig. 2). The drag curves have two positive peaks during downstroke and upstroke.

It is shown that the lift coefficients in Fig. 2a, overall, change little for different corrugation patterns during downstroke and upstroke. The first positive lift coefficient values of Corrug-1, Corrug- 2 and Corrug-3 are $0.1 \%, 10.5$ and $4.8 \%$ higher, respectively, than that of plate. For the drag coefficients, one noticeable discrepancy can be seen in Fig. 2b, the force coefficient curves have two positive peak values during the downstroke/upstroke. This finding is same as that reported by Lee and Lua [18] for flapping flight at low Reynolds numbers.

To further investigate the underlying mechanisms of the above aerodynamic effects of wing corrugations, the vorticity field around wings were provided in Fig. 4. At $\mathrm{t}=4.25 \mathrm{~T}$, it can be seen that the vorticity of the plate and corrug- 1 have the most the same strength and shape. The valley near the leading-edge was filled with vortex, and the flow field region away the leading edge have the same flow characteristics. For both the corrug-1and corrug- 2 cases, the vertex trapped in the valleys on the upper surfaces of the corrugated airfoils can be observed, as expected. There is no flow separation from the surfaces. At $\mathrm{t}=4.5 \mathrm{~T}$, there is no vortex attached to the plate, however, there is vortex remaining in the valley of the corrugated wing models. The valley was filled with vortex. One notable feature could be seen that there is a still vortices on the cambered surface. The cambered part can be seen as a large valley, which could trap the vortices. At $\mathrm{t}=4.75 \mathrm{~T}$, the flow field shows a symmetry with that at $\mathrm{t}=4.75 \mathrm{~T}$. However, the lift force coefficients show a decreasing trend for the corrugated wings. The reason may contribute the asymmetric of the wing model around the pitching axis.
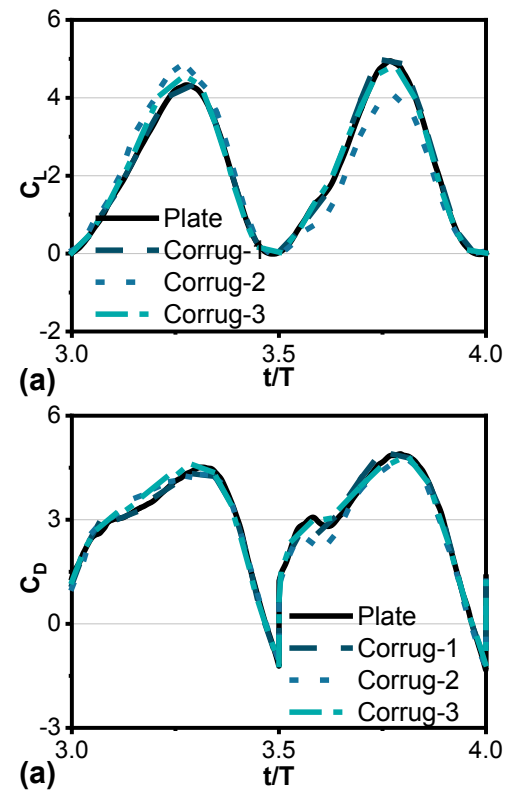

Fig. 2 The time histories of the aerodynamic forces coefficients, a) the lift coefficients and b) the drag coefficients

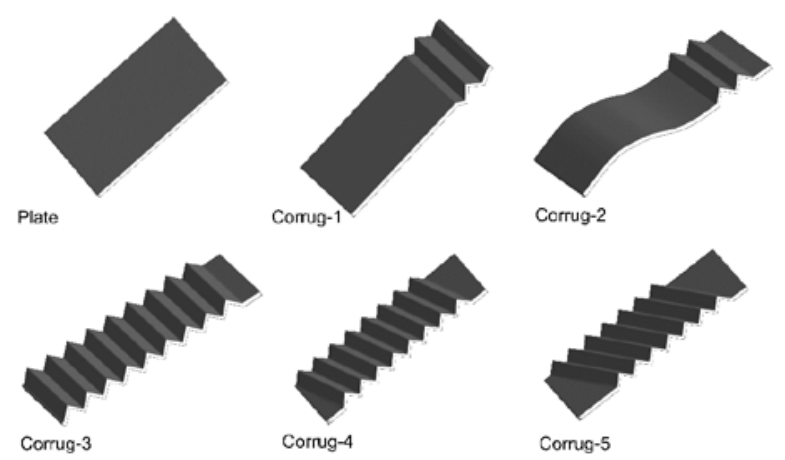

Fig. 3 The geometric sketches of the wing models 


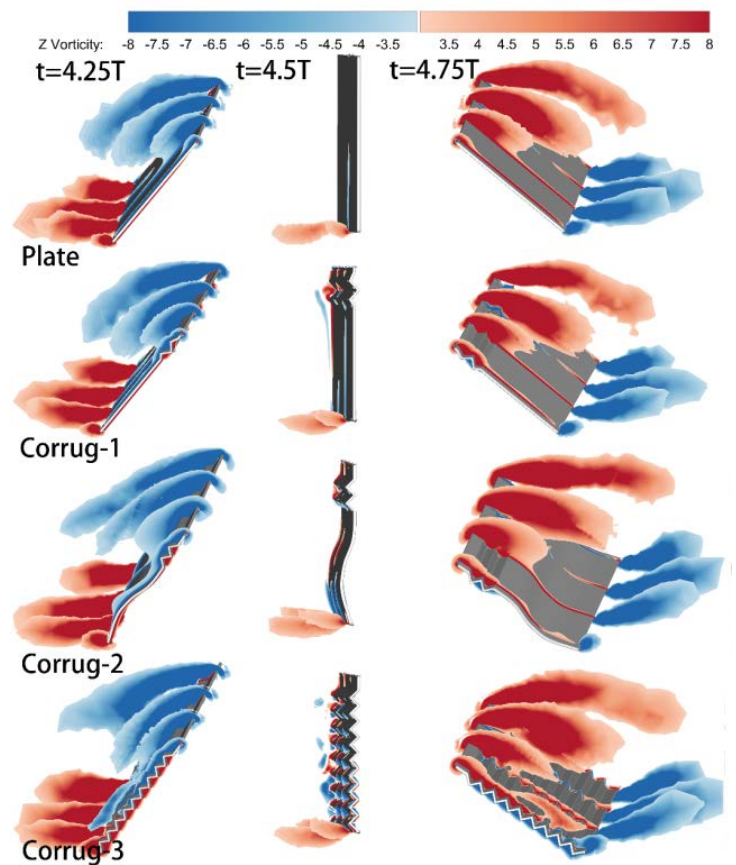

Fig. 4 The instantaneous spanwise vorticity field for different cases

\subsection{Effects of the angles of the corrugation}

The previous section discussed the effects of corrugation pattern changes. To further research the contributions of corrugation, this section focuses on a discussion about the effect of inclination angles. The different corrugated wing models are described is Fig. 3.

Overall, the lift and drag coefficient curves have similar shapes as those in the previous section (shown in Fig. 5). There are positive and negative lift coefficient peak values, while there are two positive drag coefficient peak values in the downstroke or upstroke. The peak values of the lift and drag coefficients are decreased with increasing tilt angle. The lift coefficient peak values change slightly during the upstroke. For the drag coefficients, one noticeable discrepancy can be seen, namely, the curve histories keep the same, as shown in Fig. 5.

Fig. 1 shows the pitching and plunging amplitudes for different corrugation patterns. Fig. 7 shows the time history of the aerodynamic force coefficients. The lift coefficient and drag coefficient vary with time. The results presented in Fig. 5 show that the force coefficients were significantly asymmetric during the upstroke and downstroke, which is the same with former section. The reason leads to the drag symmetrical shapes is still unclear.

Fig. 6 shows the flow field around the flapping wing in terms of the spanwise vorticity. At $t=4.25 \mathrm{~T}$, which reaches to the minimum AoA in one cycle, the wing rotates clockwise around the pitching axis and continues moving downward at the same time. At this moment, there are vortices attached to the upper surface and shed from the trailing edge into the wake. The lift and drag reach the maximum.
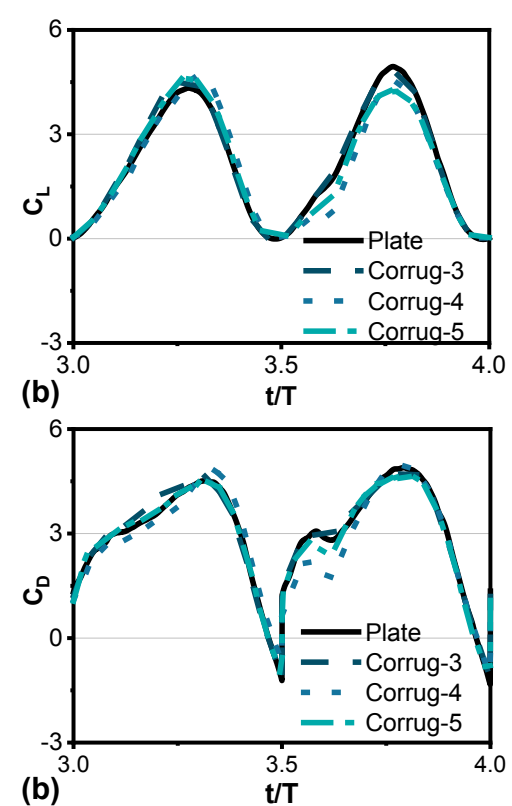

Fig. 5 The aerodynamic forces, (a) lift coefficients and (b) drag/thrust coefficients.

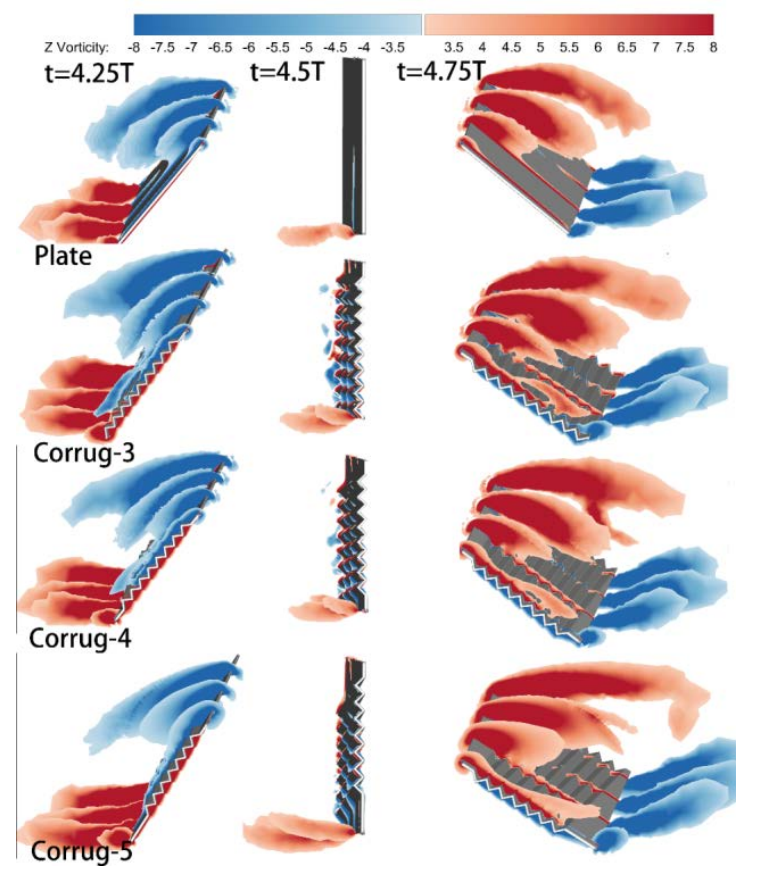

Fig. 6. The instantaneous spanwise vorticity field for different cases

At $\mathrm{t}=4.5 \mathrm{~T}$, the former LEVs and trailing edge vortex have successfully separated from the wing. Some relatively small vortices have already formed in the valley of the corrugated wing while that of flat-plate not. The following wing movement is similar to the previous movement in a timely symmetric manner; as a result, the symmetry phenomenon affects the integrated aerodynamic coefficients.

\section{Conclusion}

The effects of the wing corrugations were studied using CFD method during hovering flight at Reynolds 
numbers in the order of $10^{4}$. Different corrugation patterns and inclination angles were discussed using the instantaneous aerodynamic forces and the vorticity field around the wing models. The findings are as follows: (1) the results of this paper show that patterns of corrugation have different effect on aerodynamic performances. The corrugation like corrug- 1 changes the lift and the drag very slightly compared with a flat-plate. The corrugation patterns like Corrug- 2 and 3 of wing model reduce the lift and drag force (during the upstroke, the lift peak values, 10.5 and $4.8 \%$ lower than that of plate, respectively). (2) the increase in the inclination angle has limited effect the aerodynamic forces. The inclination angles like Corrug- 3 and 4 produce almost the same forces (the difference is less than $1 \%$ ).

\section{References}

1. Zhang, S., Y. Sunami, and H. Hashimoto, Deformation behavior of dragonfly-inspired nodus structured wing in gliding flight through experimental visualization approach. Scientific Reports, 2018. 8(1): p. 5751.

2. Shyy, W., et al., Aerodynamics, sensing and control of insect-scale flapping-wing flight. Proceedings of the Royal Society A: Mathematical, Physical and Engineering Science, 2016. 472(2186): p. 20150712.

3. Hord, K. and Y. Liang, Numerical Investigation of the Aerodynamic and Structural Characteristics of a Corrugated Airfoil. Journal of Aircraft, 2012. 49(3): p. 5 в Ђ“101.

4. Rees, C.J.C., Aerodynamic properties of an insect wing section and a smooth aerofoil compared. Nature, 1975. 258(5531): p. 141-142.

5. Newman, B.G., S.B. Savage, and D. Schonella, Model tests on a wing section of an Aeschna dragonfly, in Scale effect in animal locomotion, T.J. Pedley, Editor. 1977. p. 445-477.

6. Rudolph, R., Aerodynamic properties of Libellula quadrimaculata L.(Anisoptera: Libellulidae), and the flow around smooth and corrugated wing section models during gliding flight. Odonatologica, 1978. 7(1): p. 49-58.

7. Kesel, A.B., Aerodynamic characteristics of dragonfly wing sections compared with technical aerofoils. Journal of Experimental Biology, 2000. 203(Pt 20): p. 3125-35.
8. $\mathrm{Hu}, \mathrm{H}$. and M. Tamai, Bioinspired Corrugated Airfoil at Low Reynolds Numbers. Journal of Aircraft, 2008. 45(6): p. 2068-2077.

9. Levy, D.-E. and A. Seifert, Simplified dragonfly airfoil aerodynamics at Reynolds numbers below 8000. Physics of Fluids, 2009. 21(7): p. 071901.

10. Chen, Y.H. and M. Skote, Gliding performance of 3-D corrugated dragonfly wing with spanwise variation. Journal of Fluids and Structures, 2016. 62: p. 1-13.

11. Bomphrey, R.J., et al., Flight of the dragonflies and damselflies. Philosophical Transactions of the Royal Society B: Biological Sciences, 2016. 371(1704): p. 20150389.

12. Luo, G. and M. Sun, The effects of corrugation and wing planform on the aerodynamic force production of sweeping model insect wings. Acta Mechanica Sinica, 2005. 21(6): p. 531-541.

13. Meng, X.G., L. Xu, and M. Sun, Aerodynamic effects of corrugation in flapping insect wings in hovering flight. The Journal of Experimental Biology, 2011. 214(3): p. 432-444.

14. Flint, T., et al., Validated Unsteady Computational Fluid Dynamic Analysis of an Oscillating BioInspired Airfoil. Applied Mechanics and Materials, 2015. 799-800: p. 698-706.

15. Liu, Z., et al., Artificial insect wings with biomimetic wing morphology and mechanical properties. Bioinspiration \& Biomimetics, 2017. 12.

16. Lee, Y.J., et al., A quasi-steady aerodynamic model for flapping flight with improved adaptability. Bioinspiration \& Biomimetics, 2016. 11(3): p. 036005.

17. Lua, K.B., T.T. Lim, and K.S. Yeo, Scaling of Aerodynamic Forces of Three-Dimensional Flapping Wings. AIAA Journal, 2014. 52(5): p. 1095-1101.

18. Lee, Y.J. and K.B. Lua, Wing-wake interaction: comparison of $2 D$ and $3 D$ flapping wings in hover flight. Bioinspiration \& Biomimetics, 2018. 13(6): p. 066003.

19. Sun, M. and J. Tang, Unsteady aerodynamic force generation by a model fruit fly wing in flapping motion. Journal of Experimental Biology, 2002. 205(Pt 1): p. 55-70.

20. New, T.H., et al., Effects of Corrugated Aerofoil Surface Features on Flow-Separation Control. AIAA Journal, 2013. 52(1): p. 206-211. 